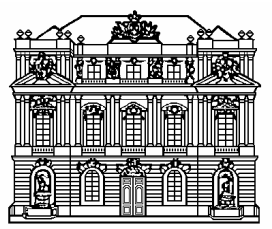

des theoretisch wissenschaftlichen Teiles

\title{
9 Verzeichnisse
}

\section{Projektwerber und Projektleitung:}

INSTITUT FÜR STRASSEN- UND VERKEHRSWESEN (ISV)

Technische Universität Graz

Rechbauerstraße 12/II, A-8010 Graz

Projektleiter: Univ.-Prof. Dr.techn. Werner GOBIET

Projektpartner:

INSTITUT FÜR ZIVILRECHT (IZR)

Universität Innsbruck

HERRY CONSULT GMBH

Em. Univ.-Prof. Dr. Peter FALLER

VERFASSER: FORSCHUNGSGRUPPE AIp-EmiV

Univ.-Prof.Dr.techn. Werner GOBIET (ISV, Projektleiter)

Em.Univ.-Prof.Dr. Peter FALLER

Dr.techn. Markus FREWEIN (ISV, Projektmanagement)

Ass.-Prof. Mag. Dr. Peter JORDAN (IZR)

Dr. Max HERRY (Herry Consult Gmbh)

unter Mitarbeit von

Martin VILHAR (ISV)

ISBN-10 3-7001-3778-8

ISBN-13 978-3-7001-3778-8

doi: 10.1553/alp-emiv

http://epub.oeaw.ac.at/alp-emiv

Graz, im Juni 2006 


\section{Verzeichnisse}

\section{$9.1 \quad$ Abkürzungen}

ABGB Allgemeines Bürgerliches Gesetzbuch, JGS Nr. 946/1811, zuletzt novelliert BGBI. I Nr. 51/2005.

Art

Artikel

BAT

best available technique(s)

BGBI

Bundesgesetzblatt

BREF

best available technique(s) reference document

BStG 1971

Bundesgesetz vom 16. Juli 1971, betreffend die Bundesstraßen (Bundesstraßengesetz 1971 - BStG 1971), BGBI. Nr. 286/1971, zuletzt novelliert BGBI. I Nr. 154/2004.

CDM: $\quad$ Clean Development Mechanism

CO2: Kohlenstoffdioxid

COP-Wert NOx-Emission eines Schwerlastkraftwagens, gemessen in $\mathrm{g} / \mathrm{kWh}$

ecolex

EU ecolex - Fachzeitschrift für Wirtschaftsrecht

FG Alp_EmiV: Forschungsgruppe Emissionsgesteuerter Alpenverkehr

ForstG 1975 Bundesgesetz vom 3. Juli 1975, mit dem das Forstwesen geregelt wird (Forstgesetz 1975), BGBI. Nr. 440/1975, zuletzt novelliert BGBI. I Nr. 87/2005.

GewO 1994 Gewerbeordnung 1994, BGBI. Nr. 194/1994 (WV)

HC: $\quad$ Kohlenwasserstoffe

idF in der Fassung von ...

IVU/IPPC-RL Richtlinie 96/61/EG des Rates vom 24. September 1996 über die integrierte Vermeidung und Verminderung der Umweltverschmutzung

$\mathrm{JBI}$

Juristische Blätter

Jgs

Josefinische Gesetzessammlung

NOx:

Stickstoffoxid

$\mathrm{OGH}$

Oberster Gerichtshof

ÖJZ

Österreichische Juristenzeitung

$\mathrm{PAH}$ :

POP

Polyzyklische Aromatische Kohlenwasserstoffe

PPP persistente organische Schadstoffe

RGBI Reichsgesetzblatt

RL Richtlinie

Rspr Rechtsprechung

StF Stammfassung

SZ Sammlung Zivilsachen (Entscheidungssammlung des Oberster Gerichtshofs)

TSP: $\quad$ Total Suspended Particles, Masse des Gesamtstaubes

WV Wiederverlautbarung

WTP willingness to pay 


\subsection{Literatur}

AICHER Josef, Grundfragen der Staatshaftung, 204ff.

ASCHWANDEN, T.; S. Cuypers; M. Fellendorf; M. Frewein; W. Gobiet; W. Hartl; G. Hauger, A. Salamon; R. Weißensteiner: Band 32 der Schriftenreihe der Institute Eisenbahnwesen und Verkehrswirtschaft, Straßen- und Verkehrswesen, Technische Universität Graz: Seminar „Verkehr im Alpenraum“, Juni 2005

BGBI. Nr. 210/1992: Bundesgesetz über Maßnahmen zur Abwehr der Ozonbelastung und die Information der Bevölkerung über hohe Ozonbelastungen, mit dem das Smogalarmgesetz, BGBI. Nr. 38/1989, geändert wird (Ozongesetz)

BGBI. I Nr. 115/1997 in der Fassung BGBI. I Nr. 34/2003: Bundesgesetz zum Schutz vor Immissionen durch Luftschadstoffe, mit dem die Gewerbeordnung 1994, das Luftreinhaltegesetz für Kesselanlagen, das Berggesetz 1975, das Abfallwirtschaftsgesetz und das Ozongesetz geändert werden (Immissionsschutzgesetz - Luft, IG-L)

BGBI. II Nr. 298/2001: Verordnung des Bundesministers für Land- und Forstwirtschaft, Umwelt und Wasserwirtschaft über Immissionsgrenzwerte und Immissionszielwerte zum Schutz der Ökosysteme und der Vegetation

BGBI. I Nr. 34/2003: Bundesgesetz, mit dem das Bundesgesetz über nationale Emissionshöchstgrenzen für bestimmte Luftschadstoffe (Emissionshöchstmengengesetz-Luft, EG-Luft) erlassen sowie das Ozongesetz und das IGLuft geändert wird

CERWENKA, Peter; Georg HAUGER; Bardo HÖRL; Michael KLAMER (2000): „Kompendium der Verkehrssystemplanung", Österreichischer Kunst- und Kulturverlag, Wien 2000

ECOPLAN/RAPP TRANS AG: Alpentransitbörse, Abschätzung der Machbarkeit verschiedener Modelle einer Alpentransitbörse für den Schwerverkehr; VSS Forschungsprojekt 2002/902; Bern/Basel 2004

FG Alp-EmiV (2005) „Anlagenrechtliche Innovationen und Emissionsrechtehandel zur Steuerung des Verkehrs in ökologisch sensiblen Gebieten - Konzentration der Analyse auf die umsetzungsrelevanten Problemschwerpunkte" (Status: nicht veröffentlicht), verfasst von der Forschungsgruppe (FG) Alp-Emiv: Faller, Frewein, Gobiet, Herry, Jordan im Auftrag der Österreichischen Akademie der Wissenschaften (ÖAW), Graz, im März 2005

FREWEIN, Markus (2005): „Der „dynamische ökologische“ Fußabdruck: Ein Beitrag zur Verminderung der Diskrepanz zwischen umweltpolitischen Zielen und Umweltwirkungen des Straßengüterverkehrs in ökologisch sensiblen 
Gebieten - gezeigt am Beispiel der NOx-Kontingentierung in einem österreichischen Alpental“, Dissertation am Institut für Straßen- und Verkehrswesen, TU-Graz

JABORNEGG Peter, Strasser Rudolf (Hrsg.), Privatrecht und Umweltschutz, 1976.

KERSCHNER Ferdinand (Hrsg.), Österreichisches und Europäisches Verkehrsrecht, 2001.

KLANG/GSCHNITZER, Kommentar zum Allgemeinen Bürgerlichen Gesetzbuch2, Bd II, 1950

KOZIOL Helmut, Österreichisches Haftpflichtrecht, Bd 2, Besonderer Teil, 1975.

RL 1996/62/EG: Richtlinie vom 27. September 1996 über die Beurteilung und die Kontrolle der Luftqualität

RL 1999/30/EG: Richtlinie vom 22. April 1999 über Grenzwerte für Schwefeldioxid, Stickstoffdioxid und Stickstoffoxide, Partikel und Blei in der Luft

RL 1999/62/EG: Richtlinie vom 17. Juni 1999 über die Erhebung von Gebühren für die Benutzung bestimmter Verkehrswege durch schwere Nutzfahrzeuge

RL 2000/69/EG: Richtlinie vom 16. November über Grenzwerte für Benzol und Kohlenmonoxid in der Luft

RUMMEL Peter (Hrsg.), Kommentar zum Allgemeinen Bürgerlichen Gesetzbuch 3, 2000.

RUMMEL Peter, Erfolgshaftung im Nachbarrecht? JBI 1997, 122.

STEINIGER Karl/GOBIET Werner et al.: Technologien und Wirkungen von PkwRoad-Pricing im Vergleich, Schriftenreihe der Institute Eisenbahnwesen und Verkehrswirtschaft, Straßen- und Verkehrswesen, Heft 29,TU Graz, 2005.

STEININGER Viktor zu OGH 26.5.1964, 8 Ob 147/64, JBI 1965, 417

THUDIUM J.; F. SIEGRIST; P. MALY (2001): „Beiträge zu einer immissionsklimatisch abgestützten Lenkung der Verkehrsströme auf der Inntalautobahn“, im Auftrag der Tiroler Landesregierung, Zürich, Juni 2001

THUDIUM J. (2002b): „Einhaltung der Grenzwerte für das NO2-Jahresmittel an der Messstelle Vomp: Szenarien der zukünftigen Entwicklung des Schweren Güterverkehrs 2002-2012“, im Auftrag der Tiroler Landesregierung, Chur, Juni 2002 
UBA, (2004): „Luftschadstoff-Trends in Österreich 1980-2004“, Umweltbundesamt $\mathrm{GmbH}$, Wien, 2004

UBA (2005): www.umweltbundesamt.at/fileadmin/site/umweltthemen/raumplanung/1 flaech..., letzter Zugriff 24.05.2005

UMWELTBUNDESAMT Deutschland [Hrsg.]: Emissionshandel im Verkehr - Ansätze für einen möglichen UP-Stream-Handel im Verkehr; Köln, Heidelberg, Mannheim, Karlsruhe, März 2005

WAGNER Erika, Die Betriebsanlage im zivilen Nachbarrecht, 1997.

WILLHELM Georg, Abgasimmissionen im Zivilrecht, ecolex 1990, 73.

Weitere Literaturhinweise sind in den Fußnoten der einzelnen Kapitel ersichtlich. 


\subsection{Abbildungen}

Abbildung 1: Organisationsschema (intern) der Forschungsgruppe AlpEmiV

Abbildung 2: Projektteile: Teil 1 Grundlagenforschung und Teil 2 angewandte Forschung

Abbildung 3: Regelkreis einer emissionsbasierten, immissionsgesteuerten Verkehrslenkung (Quelle: Frewein 2005, Abb. 9.3-2)

Abbildung 4: Bearbeitungsstand Alp-EmiV im gesamten Planungsprozess des Forschungsprojektes 30

Abbildung 5: Interaktion zwischen Fachbereichen und Systembausteinen ..............33

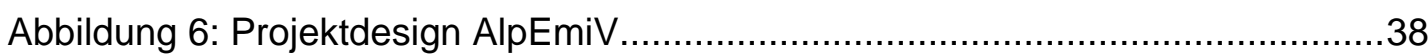

Abbildung 7: Ableitung der für das Forschungsprojekt vorrangigen Emissionengruppen 46

Abbildung 8: Kausalkette verkehrsbedingter Umweltwirkungen (Quelle Hauger 2003, Seite 144, Tabelle 30, erweitert)

Abbildung 9: $\quad$ Überblick über die Schadstoffemissionen und deren Wirkung (UBA 2004, Seite 15, Tabelle 1)

Abbildung 10: Anteile der Hauptverursacher an den

Treibhausgasemissionen (UBA 2004, Seite 20, Abb. 4) ...............52

Abbildung 11: Versauerung nach Sektoren (UBA 2004, Seite 43, Abb. 22) .............53

Abbildung 12: Anteil der Verkehrsemissionen an den Gesamtemissionen (UBA 2004, Seite 70, Abb. 48, erweitert) .54

Abbildung 13: NOx-Trend 1985 bis 2002 und Zielwerte (UBA 2004, Seite 36, Abb. 15) 55

Abbildung 14: NOx-Trend 1985 bis 2002 nach Sektoren (UBA 2004, Seite 37, Abb. 16)

Abbildung 15: CO2-Trend 1985 bis 2002 nach Sektoren (UBA 2004, Seite 27, Abb. 9) .57

Abbildung 16: PAH-Trend 1990 bis 2002 (UBA 2004, Seite 60, Abb. 38) 58

Abbildung 17: TSP-Emissionen nach Sektoren 1990, 1995, 1999, 2001 und 2002 (UBA 2004, Seite 51, Abb. 30)

Abbildung 18: Zusammenhang zwischen Zielvorstellungen, Funktionen und realisierten Nutzungen in ökologisch sensiblen Gebieten

(Quelle: Frewein 2005, Seite 78, Abbildung 4.3-1).

Abbildung 19: Überblick über den Zusammenhang von Emission -

Transmission und Immission

Abbildung 20: Mittlerer saisonaler Tagesgang des I/E-Verhältnisses an der Station Vomp 1999/2000 (Thudium 2001a, Seite 49 Abb. 4.6 tlw.) 


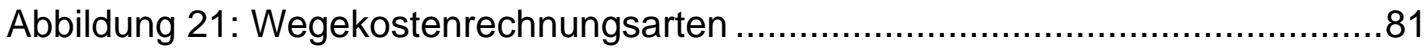

Abbildung 22: Beispiel einer Business-Account-Methode..................................83

Abbildung 23: Grundsätzliches Ablaufschema der Berechnungen zur Vollkostenrechnung

Abbildung 24: Abgangsfunktion für Autobahnen und Schnellstraßen .....................90

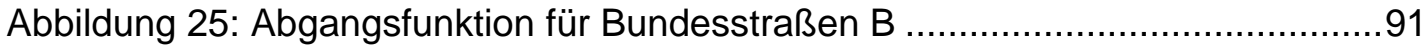

Abbildung 26: Abgangsfunktionen der Landes- und Gemeindestraßen .................91

Abbildung 27: Prinzipielle Ordnung zwischen Lenkungsmechanismen und

Lenkungsinstrumenten mit Fokus auf umweltorientierte

Lenkungsinstrumente.

Abbildung 28: Modellierung als Zwischenschritt bei der Suche nach einer realen Problemlösung (Vgl. Müller-Merbach, Heiner, Operations Research, 2. Aufl., München 1971, S. 14).

\subsection{Tabellen}

Tabelle 1: $\quad$ Zuteilung der einzelnen Fachbereiche zu den

Projektpartnern (Hauptverantwortlichkeiten). 12

Tabelle 2: $\quad$ Mitglieder des Fachbeirates Alp-Emiv

Tabelle 3: Komponenten für das Emissionsrechtehandelssystem im

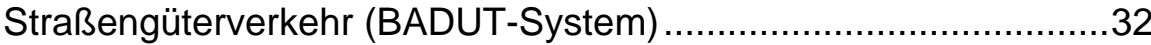

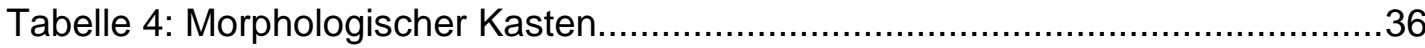

Tabelle 5: Beschreibung der Merkmale und Ausprägungen ...................................37

Tabelle 6: $\quad$ Emissionsquellengruppen mit dem Lokalisierungstyp (Quelle: Brauer (Hrsg.) 1996, Seite 58 (Tabelle 2.1) erweitert)

Tabelle 7: $\quad$ Überblick über die Entwicklungstendenzen von für das Forschungsprojekt relevanten Schadstoffemissionen (Datenquelle: UBA 2004)

Tabelle 8: $\quad$ Für das Forschungsprojekt relevante verkehrlich bedingte Schadstoffemissionen unterteilt nach Relevanz durch normative Festlegungen und Trendentwicklung..........................70

Tabelle 9: Lebensdauern für Autobahnen nach WKR 000 ........................................8

Tabelle 11: $\quad$ Allgemeine projektbezogene Forschungsthemen (Grundlegende Ansatzpunkte) 107

Tabelle 12: Gebietsbezogene Forschungsthemen......................................109

Tabelle 13: Infrastrukturbezogene Forschungsthemen ................................110

Tabelle 14: $\quad$ Lenkungsinstrument: Entwicklungsbezogene

Forschungsthemen 
Tabelle 15: Lenkungsinstrument: Entwicklungsbezogene

Forschungsthemen, die sich noch in Diskussion befinden

Tabelle 16: Lenkungsinstrument: Umsetzungsbezogene

Forschungsthemen, die sich noch in Diskussion befinden

Tabelle 17: Verkehrslenkungsmechanismen unterteilt nach Gesellschafts- und Umfeldbereichen (nach Cerwenka et al. 2000, stark erweitert)

Tabelle 18: Umweltorientierte Verkehrslenkungsinstrumente im Straßengüterverkehr nach Umfeldbereichen geordnet (Quelle: Cerwenka/Hauger stark erweitert und verändert)

Tabelle 19: $\quad$ Überblick über die wesentlichen und für das Forschungsprojekt relevanten normativen Festlegungen von Grenzwerten von Luftschadstoffen

Tabelle 20: Zusammenstellung der angewandten Untersuchungsmethoden für den Fachbereich Verkehrsplanung

Tabelle 21: Zusammenstellung der angewandten und diskutierten Untersuchungsmethoden für den Fachbereich Verkehrsökologie.

Tabelle 22: Zusammenstellung der angewandten und diskutierten Untersuchungsmethoden für den Fachbereich Verkehrsrecht und rechtliche Grundlagen

Tabelle 10: Externe Kosten 2000 für Fahrzeuge über 3,5 t hzG auf Autobahnen und Schnellstraßen in Österreich 